\title{
PRÉVENTION
}

\section{Nutrition \& cancer, controverses et vérités}

Face à la multiplication récente de dogmes alimentaires anti cancer parfois complètement infondés, la toute nouvelle Agence de sécurité sanitaire (Anses) s'est autosaisie du dossier Nutrition \& cancer et vient de rendre ses conclusions. S'il n'existe aucun aliment anti-cancer à proprement dit, une prévention nutritionnelle réelle est possible.

\section{/// Se gaver de brocoli et de} jus de grenade, bannir la côte de bœuf et les produits laitiers... Alors que se multiplient de façon exponentielle les régimes miracles pour perdre du poids, on assiste en parallèle à la naissance d'une nouvelle discipline dans le domaine de la nutrition, celles des régimes anticancer. Des plus sérieux aux plus dangereux, ces messages laissent parfois le grand public dans un état de désarroi, et peut même culpabiliser certains malades, leur laissant croire qu'ils n'ont pas pris les mesures nécessaires pour éviter leur cancer. C'est pourquoi l'Agence française de sécurité sanitaire des aliments (Afssa) s'est saisie de la question dès 2007. Après quatre ans de travaux, I'Agence de sécurité sanitaire (Anses, issue de la fusion de l'Afssa et de l'Afsset) a publié au printemps dernier un rapport ${ }^{1}$ dans lequel elle affirme : "On dispose aujourd'hui d'un faisceau d'arguments montrant que la prévention nutritionnelle des cancers est légitime et qu'elle peut être efficace. »

\section{Pas d'aliment ni de régime anti-cancer}

Pour autant il n'y a pas de solution miracle. Le rapport de l'Anses est très clair : "L'expression "aliment anti-cancer" est trompeuse : elle peut laisser croire que la consommation d'un aliment en particulier peut empêcher le développement d'un cancer, voire le guérir, ce qui est totalement faux et infondé. En effet, aucun aliment, quelle que soit sa composition nutritionnelle, ne peut, à lui seul, s'opposer au développement d'un cancer ou de toute autre maladie. » Voilà qui devrait faire réfléchir les fervents défenseurs du thé vert et du curcuma, parmi lesquels les plus éminents cancérologues... L'Agence va même plus loin en affirmant que l'expression « régime anti cancer est fausse, scientifiquement infondée, voire dangereuse. En effet, aucun régime alimentaire ne peut se substituer à un traitement médical, quelle que soit la maladie.»

En cela, I'Anses se réfère largement à l'expertise ${ }^{2}$ conduite en
2006 par l'American Institute for Cancer Research (IACR) et le Fonds international de recherche contre le cancer (WCRF) : «Du point de vue de la prévention des cancers, il est nécessaire de raisonner de manière globale et ainsi, seule une alimentation équilibrée et diversifiée avec un apport calorique adapté aux dépenses énergétiques jointe à une activité physique régulière peut contribuer à réduire le risque $»$. De fait, selon le rapport IACR/WCRF, « un tiers des cancers les plus communs pourraient être évités dans les pays industrialisés grâce à la prévention nutritionnelle.»

Tableau : Huit facteurs « convaincants ».

Dans son rapport, I'Anses pointe du doigt huit facteurs avec « des niveaux de preuve convaincants ou probables de relation avec le cancer ».

\begin{tabular}{|c|c|c|}
\hline Aliments/conduites & $\begin{array}{l}\text { Facteurs de risques } \\
\text { convaincants }\end{array}$ & $\begin{array}{c}\text { Facteurs de risques } \\
\text { probables }\end{array}$ \\
\hline Boissons alcoolisées & $\begin{array}{l}\text { Cancers de la bouche, du pharynx, } \\
\text { du larynx, de l'œsophage, } \\
\text { colorectal chez I'homme }\end{array}$ & $\begin{array}{l}\text { Cancer colorectal chez la femme } \\
\text { Cancer du foie }\end{array}$ \\
\hline Surpoids et obésité & $\begin{array}{l}\text { Cancers de l'œesophage, de } \\
\text { l'endomètre, du rein, colorectal, } \\
\text { du pancréas, du sein après la } \\
\text { ménopause }\end{array}$ & - \\
\hline Viandes rouges et charcuteries & Cancer colorectal & - \\
\hline Sels et aliments salés & - & Cancer de l'estomac \\
\hline $\begin{array}{l}\text { Compléments alimentaires de } \\
\text { type bêtacarotène }\end{array}$ & $\begin{array}{l}\text { Cancer du poumon chez les } \\
\text { fumeurs }\end{array}$ & - \\
\hline Aliments/conduites & $\begin{array}{l}\text { Facteurs de prévention } \\
\text { convaincants }\end{array}$ & $\begin{array}{l}\text { Facteurs de prévention } \\
\text { probables }\end{array}$ \\
\hline Activité physique & Cancer du côlon & Nombreux cancers courants \\
\hline Fruits et légumes & - & $\begin{array}{l}\text { Cancers de la bouche, du pharynx, } \\
\text { du larynx, de l'œesophage, de } \\
\text { l'estomac, du poumon }\end{array}$ \\
\hline $\begin{array}{l}\text { Allaitement exclusif jusqu'à } \\
6 \text { mois }\end{array}$ & Cancer du sein & - \\
\hline
\end{tabular}

\section{Des messages clairs}

L'Anses estime que les huit facteurs faisant actuellement l'objet de recommandations nutritionnelles sont pertinents pour la population française (voir tableau). Prises dans leur globalité, ces recommandations permettent d'identifier trois objectifs prioritaires. Il s'agit de : - réduire la consommation d'alcool ;

- promouvoir une alimentation équilibrée et diversifiée ;

- promouvoir la pratique d'une activité physique régulière.

Pour aller plus loin, et surtout aider les professionnels face aux questions que se posent les malades mais aussi le grand public, une série de questions-réponses qui rappelle les messages essentiels et clarifie certaines idées fausses a été élaborée ${ }^{3}$. Elle aborde des thèmes très divers : consommation de viandes et charcuteries, d'épices et plantes aromatiques, une alimentation riche en fibres ou en matières grasses, un apport supplémentaire en vitamines ou antioxydants... Elle fait aussi le point sur les risques associés au surpoids, sur les modes d'action des principaux facteurs nutritionnels sur le risque de cancer, sur I'allaitement, le mode de cuisson des aliments...

Emilie Gillet

$\nabla$ POUR EN SAVOIR PLUS

1 Sur le site de l'Anses le rapport 2011 « Nutrition et cancer » : www.anses.fr/Documents/ NUT2007sa0095Ra.pdf

2 Site dédié au rapport WCRF/IACR « Food, Nutrition, Physical Activity and the Prevention of Cancer:a Global Perspective » version 2010 : www.dietandcancerreport.org/

320 questions réponses pratiques sur les rapports entre nutrition et cancer : www.anses.fr/Documents/ NUT-QR-NutritionCancer.pdf 